\title{
Un nouveau type de globine fonctionnel chez les primates?
}

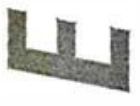

\section{Les nouvelles de ce numéro ont été préparées par : J.-C. Dreyfus}

\section{A. Kahn}

1. Marks J, Shaw JP, Chen CKJ. Sequence organization and genomic complexity of primate $\theta_{1}$ globin gene, a novel $\alpha$-globin-like gene. Nalure $1986 ; 321$ : 785-8.

2. Shaw JP, Marks J, Chen CKJ. Evidence that the recently discovered $\theta_{1}$ gene is functional in higher primates. Nature 1987 ; 326 : 717-20.

3. Cheng JF, Raid L, Hardison RC. Isolation and nucleotide sequence of the rabbit gene cluster $\psi \zeta-\alpha_{1}-\psi \alpha_{1}$.J Biol Chem 1986; 261 : 839-48.

4. Sawada I, Schmid CW, Primate evolution of the $\alpha$-globin gene cluster and its Alu-like repeats. J Mol Biol 1986; 192 : 693-709.

$\mathrm{m} / \mathrm{s} n^{\circ} 7$ vol. 3, septembre 87
Les gènes de globine des mammiferes sont regroupés dans deux régions, situées chez l'homme sur les chromosomes 16 pour les gènes $\alpha$ et 11 pour les gènes $\beta$. Sur le chromosome 16 se trouvent les gènes $\alpha$, et $\alpha_{2}$, ainsi que le gène embryonnaire $\zeta$. De plus, on connaît des pseudogènes de globine non fonctionnels car truffés de codons de terminaison. En 1986 Marks et al. (Davis, California) ont découvert un nouveau type de gène $\alpha$ chez plusieurs espèces de mammiferres [1]. $\mathrm{Ce}$ gène, appelé $\theta$, est situé $3 \mathrm{~kb}$ seulement en aval de $\alpha$. Il a été cloné et sa séquence déterminée chez deux espèces de primates, l'orang-outan (Pongo Pygmaeus) et le babouin olive (Papio Anubis) [2]. Dans les deux cas, il existe une phase ouverte de lecture, codant pour une chaîne de 141 acides aminés comme pour les chaînes $\alpha$ et $\zeta$; des signaux "promoteur " et d'épissage sont présents. D'autres arguments, tirés de l'évolution, plaident en faveur du caractère fonctionnel du gène : les différences entre les nucléotides d'orang-outan et de babouin sont, pour le gène $\alpha$, de 26 dont 12 conduisent à un changement d'acide aminé ; pour le gène $\theta$, elles sont respectivement de 27 et seulement 5 : il y a donc tendance au maintien de la structure protéique. La région promotrice est également conservée d'une espèce à l'autré.

Il existe ceperidant des dissonances qui conduisent à poser de nouvelles questions. Les séquences promotrices sont nettement plus en amont du codon initiateur qu'il n'est habituel ; aucune preuve que le gène soit fonctionnel n'a pu être apportée, la recherche du messager et les tentatives de transfection du gène étant restées infructueuses jusqu'à présent. Enfin, les résultats des comparaisons entre espèces sont troublants : un gène correspondant à $\theta$ a été trouvé chez de nombreux mammifères; mais chez le lapin [3] et un lémurien, galago [4], il a subi des modifications qui l'inactivent nécessairement. On a tenté d'interpréter l'ensemble des résultats en fonction de l'évolution: sur les 141 acides aminés de la chaîne, 55 different entre $\alpha$ et $\theta$. Selon les modes de calcul, on fait dater la séparation des deux gènés de 100 à 260 millions d'années, l'inactivation chez le lapin remontant à environ 30 millions d'années. Pourquoi un gène serait-il resté fonctionnel chez les primates supérieurs (y compris probablement l'homme, dont l'organisation de $\theta$ est identique à celle de l'orangoutan) et non chez d'autres maminiferes?

Les auteurs formulent deux hypothèses : ou bien le mode de placentation développé par les primates supérieurs réclamerait le fonctionnement de ce gène, ou bien, à l'inverse, des gènes fonctionnels non encore découverts existeraient en d'autres parties du génome, chez le lapin par exemple.

Au total, l'ensemble des données milite en faveur d'un nouveau gène de globine, qui serait fonctionnel chez les primates supérieurs ; reste à reconnaître dans quels tissus, et à quel stade du développement. La découverte de gènes dont l'action est indispensable à des stades très transitoires de l'ontogenèse justifie cette hypothèse, même si elle peut s'avérer difficile à vérifier.

J.-C. D.
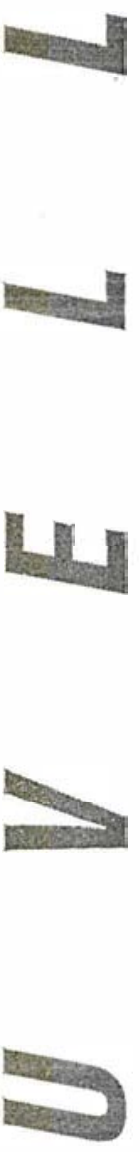